\title{
Cell cycle-dependent regulation of extra-adrenal glucocorticoid synthesis in murine intestinal epithelial cells
}

\author{
Atanas G. Atanasov,* Dominic Leiser,* Corinne Roesselet,* Mario Noti,* \\ Nadia Corazza, ${ }^{*}$ Kristina Schoonjans, ${ }^{\dagger}$ and Thomas Brunner* \\ *Division of Immunopathology, Institute of Pathology, University of Bern, Bern, Switzerland; \\ and ${ }^{\dagger}$ Institut de Génétique et de Biologie Moléculaire et Cellulaire, CNRS/INSERM/ULP, \\ Illkirch, France
}

ABSTRACT Glucocorticoids are anti-inflammatory steroids with important applications in the treatment of inflammatory diseases. Endogenous glucocorticoids are mainly produced by the adrenal glands, although there is increasing evidence for extra-adrenal sources. Recent findings show that intestinal crypt cells produce glucocorticoids, which contribute to the maintenance of intestinal immune homeostasis. Intestinal glucocorticoid synthesis is critically regulated by the transcription factor liver receptor homologue-1 (LRH-1). As expression of steroidogenic enzymes and LRH-1 is restricted to the proliferating cells of the crypts, we aimed to investigate the role of the cell cycle in the regulation of LRH-1 activity and intestinal glucocorticoid synthesis. We here show that either pharmacological or molecular modulation of cell cycle progression significantly inhibited expression of steroidogenic enzymes and synthesis of glucocorticoids in intestinal epithelial cells. Synchronization of intestinal epithelial cells in the cell cycle revealed that expression of steroidogenic enzymes is preferentially induced at the $G_{1} / S$ stage. Differentiation of immature intestinal epithelial cells to mature nonproliferating cells also resulted in reduced expression of steroidogenic enzymes. This cell cycle-related effect on intestinal steroidogenesis was found to be mediated through the regulation of LRH-1 transcriptional activity. This mechanism may restrict intestinal glucocorticoid synthesis to the proliferating cells of the crypts.-Atanasov, A. G., Leiser, D., Roesselet, C., Noti, M., Corazza, N., Schoonjans, C., Brunner, T. Cell cycle-dependent regulation of extra-adrenal glucocorticoid synthesis in murine intestinal epithelial cells.

Key Words: liver receptor homologue-1 $\cdot$ nuclear receptors $\cdot$ steroidogenesis $\cdot$ mucosal immunology

GLUCOCORTICOIDS PLAY AN IMPORTANT ROLE in regulation of immune cells and inflammation. Consequently, glucocorticoids and synthetic derivatives are successfully used in the treatment of inflammatory diseases, including inflammatory bowel disease (IBD), such as Crohn's disease and ulcerative colitis (1). The adrenal glands are the major source of endogenous glucocorticoids, and lack of endogenous glucocorticoid synthesis, e.g., after adrenalectomy, may result in shock and lethality on strong immune cell stimulation (2). Recent findings, however, support the idea that glucocorticoid synthesis is not restricted to the adrenal glands but may occur in a variety of tissues, including thymus, brain, skin, and endothelium. We have recently reported that the intestinal epithelium is also an important extra-adrenal source of immunoregulatory glucocorticoids. The enzymes required for the synthesis of glucocorticoids from cholesterol were found to be preferentially expressed in the proliferating epithelial cells of the crypt region. The synthesis of glucocorticoids in intestinal epithelial cells was induced after immune cell activation and had in turn a profound immunoregulatory effect on the activation of local T cells (3).

Glucocorticoid synthesis in the adrenal glands is critically controlled by the nuclear receptor steroidogenic factor-1 (SF-1, NR5A1) (4). SF-1 is a transcription factor that controls the transcription and expression of a variety of steroidogenic enzymes in the adrenal glands. Consequently, the production of systemic glucocorticoids is severely impaired in SF-1-deficient mice (4). SF-1 is not expressed in the intestinal epithelium and does not participate in the regulation of intestinal glucocorticoid synthesis (5). Interestingly, a close homologue of SF-1, liver receptor homologue-1 (LRH-1, NR5A2) seems to functionally substitute SF-1 in the intestinal epithelium. LRH-1 is expressed at high levels in intestinal crypt cells $(6,7)$, and overexpression of LRH-1 induces expression of steroidogenic enzymes and glucocorticoid synthesis $(5,8$, 9). In addition, LRH-1 haploinsufficient mice have strongly reduced intestinal glucocorticoid synthesis (5, 10). More recently, it was found that LRH-1 haploinsufficient mice or mice with intestinal epithelium-specific LRH-1 deficiency are more sensitive to induction of experimental colitis, supporting the notion that intestinal glucocorticoids have an important role in the regulation 
of local immune responses (10). Interestingly, it was also observed that LRH-1 expression was significantly reduced in inflamed intestinal tissue specimens of patients with Crohn's disease or ulcerative colitis compared with noninflamed control tissues (10).

LRH-1 is involved not only in the regulation of steroidogenesis but also in cell cycle control and tumor formation. LRH-1 regulates the expression of cyclin D1 and E1 in intestinal crypt cells, thereby contributing to the renewal of the intestinal epithelial layer (7). Uncontrolled LRH-1 activity, however, seems also to participate in the induction of intestinal tumors, probably through enhanced expression of cyclin proteins (6).

Here we show not only that LRH-1 controls cell cycle progression in intestinal epithelial cells but also that regulation of LRH-1 activity and associated expression of steroidogenic enzymes is cell cycle-dependent. Inhibition of cyclin-dependent kinase (Cdk) 1 or cell cycle progression by pharmacological inhibitors severely impaired LRH-1-mediated expression of steroidogenic enzymes and glucocorticoid synthesis. Interestingly, cell cycle arrest did not alter the nuclear localization of LRH-1 but reduced its transcriptional activity. In addition, both steroidogenic enzymes CYP11A1 (side chain cleavage enzyme) and CYP11B1 (11ß-hydroxylase) are preferentially induced in the $G_{1} / S$ phase of cell cycle-synchronized cells. In combination, our results suggest that in the intestine LRH-1-induced steroidogenesis is cell cycle stage-dependent.

\section{MATERIALS AND METHODS}

\section{Cells and reagents}

The murine intestinal epithelial cell line mICcl2 (11) was described previously. The culture medium consisted of $12 \mathrm{~g} / \mathrm{L}$ Dulbecco's modified Eagle medium/Ham's F-12 (1:1, v/v; Invitrogen, Carlsbad, CA, USA), $2.438 \mathrm{~g} / \mathrm{L} \mathrm{NaHCO}_{3}, 2 \%$ steroidfree fetal calf serum (FCS), $60 \mathrm{nmol} / \mathrm{L}$ sodium selenite, $5 \mu \mathrm{g} / \mathrm{ml}$ apotransferrin, $10 \mathrm{ng} / \mathrm{ml}$ murine epithelial growth factor, 1 $\mathrm{nmol} / \mathrm{L}$ triiodothyronine, $5 \mu \mathrm{g} / \mathrm{ml}$ insulin, $2 \mathrm{mmol} / \mathrm{L}$ L-alanylL-glutamine, $50 \mu \mathrm{g} / \mathrm{ml}$ gentamicin, and $20 \mathrm{mmol} / \mathrm{L}$ HEPES. Forskolin was obtained from Calbiochem (VWR, Lucerne, Switzerland). Aphidicolin, doxorubicin, olomoucine, iso-olomoucine, and nocodazole were from Alexis (Lausen, Switzerland).

\section{Plasmids}

The expression construct for murine LRH-1 (12) and the murine CYP11A1 and CY11B1 reporter constructs (5) were described earlier (12). The heterologous LRH-1 reporter construct, referred to as LRH-1 RE 5x, was generated by subcloning five copies of the LRH-1 response element (RE) of the SR-B1 promoter (13) into basic pGL3 (Promega, Duebendorf, Switzerland). An expression plasmid for green fluorescent protein (GFP)-tagged LRH-1 was generated by cloning murine LRH-1 into pEGFP-N1 (Clontech, Saint-Germain-en-Laye, France). The activities of untagged and tagged LRH-1 were tested in a luciferase reporter assay (see Fig. $6 A$ ). The pRb (retinoblastoma protein) expression plasmid was kindly provided by Seamus Martin (Trinity College, Dublin, Ireland) (14); Cdk1 and dominant negative Cdk1 expression plasmids were a gift from Ed Harlow (Massachusetts General Hospital Cancer Center, Charlestown, MA, USA) (15).
CYP11A1, CYP11B1, or LRH-1 RE 5x reporter constructs and $\beta$-galactosidase expression vector for transfection control were cotransfected into mICcl2 cells using the calcium phosphate precipitation method. In some experiments cells were cotransfected with empty vector or LRH-1, Cdk1, dominant negative $\mathrm{Cdk} 1$, and/or pRb expression plasmids. After overnight transfection, cells were washed, treated with the indicated inhibitors, and cultured for $16 \mathrm{~h}$. Cells were then lysed, and luciferase activity assays and normalization with $\beta$-galactosidase activity were performed as described previously (16).

\section{Measurement of corticosterone}

mICcl2 cells were transfected and stimulated as indicated. After overnight culture, cell-free supernatant was harvested, and corticosterone production was measured by RIA (3).

\section{Cell cycle release experiments}

mICcl2 cells were treated with control medium or $100 \mathrm{ng} / \mathrm{ml}$ nocodazole for $16 \mathrm{~h}$ to induce cell cycle arrest in the $\mathrm{G}_{2}$ phase (17). Cells were then washed two times with PBS and resuspended in medium without nocodazole. After $0,2,4,6$, and $12 \mathrm{~h}$ an aliquot of the nocodazole-treated cells or control cells was harvested and the DNA content, as a measurement of cell cycle progression, was analyzed by propidium iodide staining (17). At the same time points, aliquots were collected, and total RNA was isolated. LRH-1, cyclin B1, cyclin D1, CYP11A1, and CYP11B1 mRNA expression was analyzed by real-time reverse transcriptase (RT)-polymerase chain reaction (PCR) using QuantiTect primers (Qiagen, Basel, Switzerland) and glyceraldehyde-3-phosphate dehydrogenase mRNA as an internal control (9).

\section{Detection of steroidogenic factors in differentially isolated intestinal epithelial cells}

Alkaline phosphatase staining and differential isolation of epithelial cells from the small intestine of C57BL/6 mice were done as described previously (3). Epithelial cell fractions were analyzed for their protein content and alkaline phosphatase activity. The early fractions with a high alkaline phosphatase activity were considered villus epithelial cell fractions, whereas the late fractions with a low alkaline phosphatase activity were considered crypt cell fractions. LRH-1, cyclin B1, and CYP11B1 mRNA expression in these fractions was determined as described previously $(5,9)$.

\section{Subcellular localization of LRH-1}

mICcl2 cells were transfected with GFP-tagged LRH-1 expression plasmid and then treated with various cell cycle inhibitors at concentrations previously tested to block LRH-1-induced CYP11A1 promoter activity. The subcellular localization was then assessed using fluorescence microscopy. Alternatively, cells were transfected with a control or LRH-1 expression vector and treated with vehicle or doxorubicin. Cytoplasmic and nuclear fractions were isolated as described previously (18), and the presence or absence of LRH-1 in these fractions was assessed by Western blotting (19).

\section{Epithelial cell differentiation experiment}

Young adult mouse colon (YAMC) cells (20) were kindly provided by Robert Whitehead (Vanderbilt University, Nashville, TN, USA) and were grown in RPMI 1640 medium containing 
$5 \% \mathrm{FCS}, 50 \mu \mathrm{g} / \mathrm{ml}$ gentamicin, $1 \mu \mathrm{g} / \mathrm{ml}$ insulin, and $0.5 \mathrm{ng} / \mathrm{ml}$ murine IFN $\gamma$. YAMC cells are conditionally immortalized by an IFN- $\gamma$-inducible and temperature-sensitive simian virus 40 large $\mathrm{T}$ antigen (SV40LT) mutant (tsA58), which is active at $33^{\circ} \mathrm{C}$ and inactive at $37^{\circ} \mathrm{C}$. A shift to $37^{\circ} \mathrm{C}$ leads to a time-dependent differentiation of the crypt-like cells to mature nonproliferating epithelial cells. YAMC cells were either grown at $33^{\circ} \mathrm{C}$ or shifted to $37^{\circ} \mathrm{C}$ for various periods of time in the absence of murine IFN- $\gamma$. Proliferation of cells was assessed using the 3-(4,5-dimethylthiazol-2-yl)-2,5-diphenyltetrazolium bromide (MTT) assay (21). Cell cycle arrest was measured by staining DNA with propidium iodide and analysis by flow cytometry (17). Expression of the Cdk inhibitor p21/waf, cyclin D1, LRH-1, and CYP11B1 was measured by real-time RT-PCR.

Statistical analysis

In some experiments, differences between groups were analyzed by unpaired two-tailed Student's $t$ test. Values of $P<0.05$ were considered significant.

\section{RESULTS}

LRH-1 and steroidogenic enzymes are coexpressed in the proliferating cells of the small intestinal crypts

We have shown previously that the intestinal epithelium is an important source of extra-adrenal glucocorticoids and that the regulation of glucocorticoid synthesis in the intestinal epithelium is substantially different from that in the adrenal glands $(5,9)$. Interestingly, we found that the expression of steroidogenic enzymes in the intestine is confined to the proliferating intestinal crypt cells. The expression of steroidogenic enzymes and the synthesis of glucocorticoids in intestinal epithelial cells is critically regulated by the nuclear receptor and transcription factor LRH-1 (5). However, LRH-1 regulates not only intestinal steroidogenesis but also crypt cell proliferation and renewal (7).

We therefore hypothesized that LRH-1 and steroidogenic enzymes colocalize in the proliferating cells of the intestinal crypts. Using a differential isolation method (3), we isolated intestinal epithelial cells either from the villus tip or the intestinal crypts. Villus cells were identified by the presence of high alkaline phosphatase activity, whereas crypt cell fractions had only minimal enzyme activity (Fig. $1 A, B)$. Villus tip or crypt cell fractions were then analyzed for the expression of LRH-1, cyclin D1 as a marker for proliferating crypt cells, and CYP11B1 as a representative steroidogenic enzyme and LRH-1 target. Intriguingly, it was found that all three gene products tested colocalized in the same cell fractions, i.e., the proliferating and alkaline phosphatase-negative cells of the crypts (Fig. $1 B$ ).

Intestinal epithelial cell differentiation induces cell cycle arrest and blocks expression of steroidogenic enzymes

As epithelial cells from the villus tip were found to be negative for LRH-1 and CYP11B1 expression, we hypothesized that the differentiation of immature proliferating

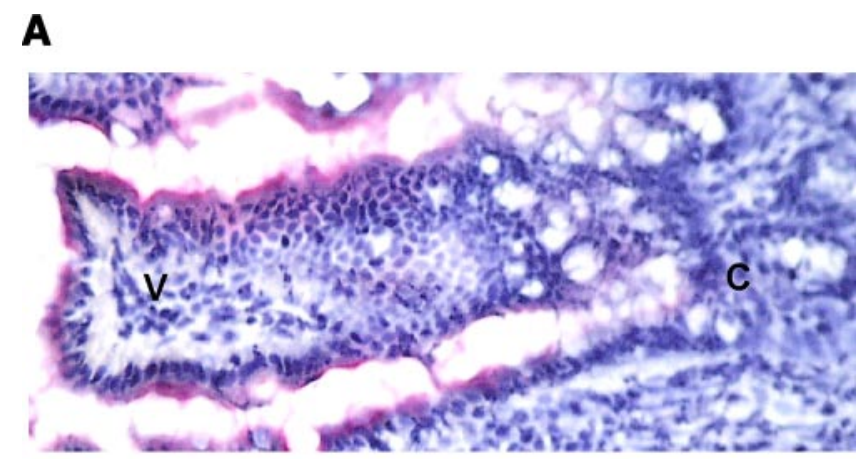

B
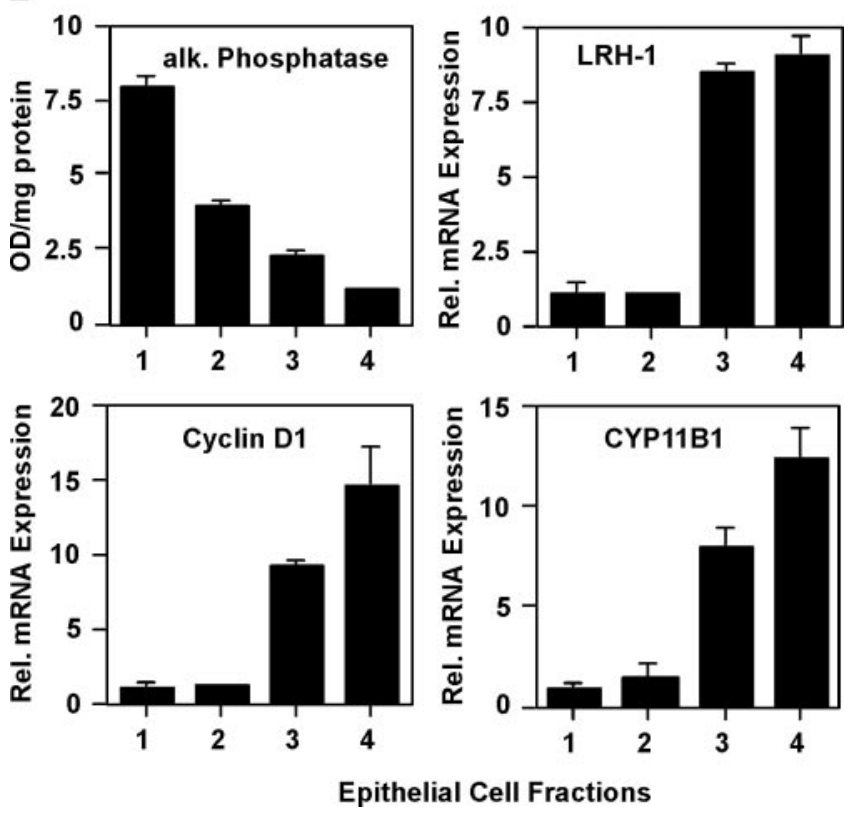

Figure 1. Colocalization of LRH-1, cyclin D1, and CYP11B1 in the intestinal crypts. A) Detection of alkaline phosphatase activity (red) in tissue sections from the small intestinal mucosa. V, villus; C, crypt. B) Intestinal epithelial cells from the small intestine were differentially isolated from the villus tip (fraction 1) to the crypt (fraction 4). Alkaline phosphatase activity (alk. phosphatase) and LRH-1, cyclin D1, and CYP11B1 expression in the different fractions are shown. A typical experiment of three is shown. Rel., relative.

crypt cells to mature differentiated epithelial cells leads to reduced expression of steroidogenic enzymes. To test this idea we took advantage of the YAMC epithelial cells, which are transformed by a temperature-sensitive SV40LT mutant (20). Although these cells grow at $33^{\circ} \mathrm{C}$, transfer to $37^{\circ} \mathrm{C}$ leads to rapid inactivation of SV40LT and differentiation of the YAMC cells. Transfer of YAMC cells to $37^{\circ} \mathrm{C}$ resulted in reduced proliferation, as measured by MTT assay (Fig. 2A) and induction of cell cycle arrest (Fig. $2 B)$. Accordingly, cyclin D1 expression was reduced in differentiated cells whereas the expression of the cell cycle inhibitor $\mathrm{p} 21 /$ Waf was induced (Fig. 2C). Interestingly, although LRH-1 expression was only partially inhibited, CYP11B1 expression was almost completely abrogated in cells grown at $37^{\circ} \mathrm{C}$ for $24 \mathrm{~h}$ (Fig. 2C). Thus, these data suggest that the expression of steroidogenic enzymes is restricted to undifferentiated proliferating epithelial cells. 


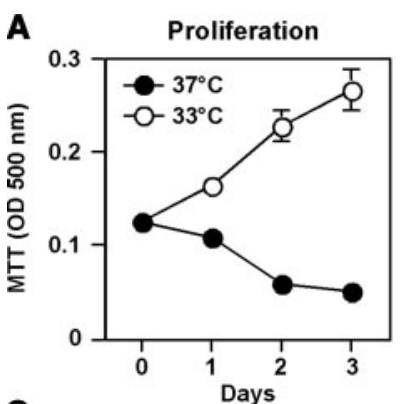

Cell cycle
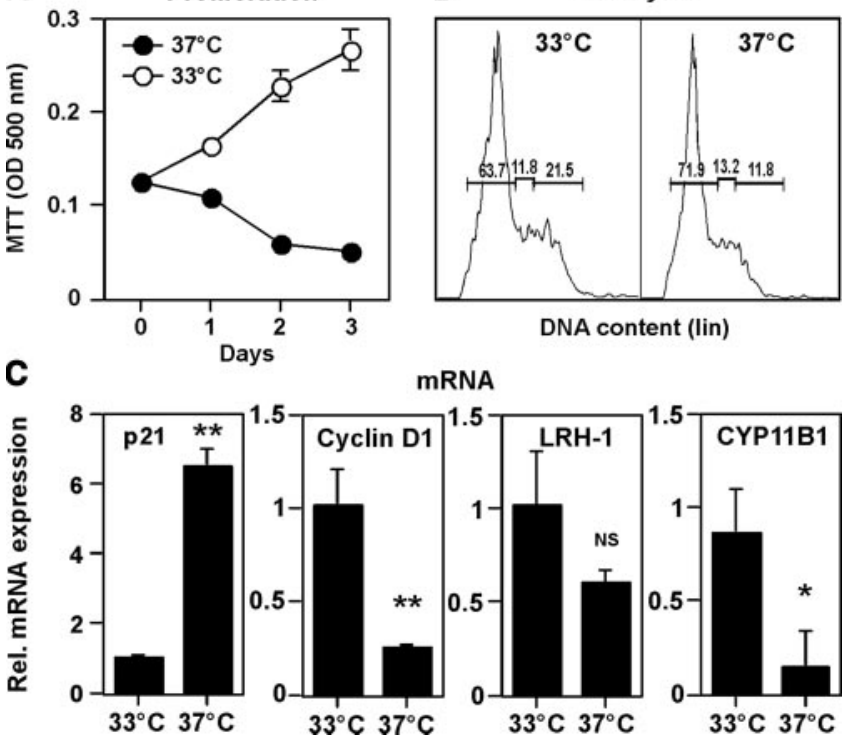

mRNA

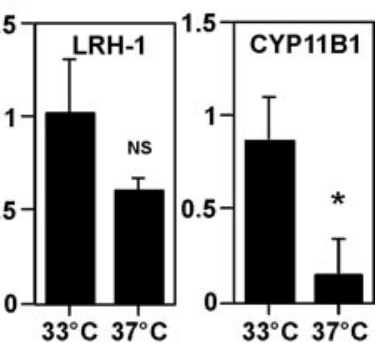

Figure 2. Epithelial cell differentiation induces cell cycle arrest and inhibits steroidogenic enzyme expression. A) YAMC cells were grown at either 33 or $37^{\circ} \mathrm{C}$ for indicated time points, and proliferation was measured by the MTT assay. B) Cells were grown at 33 or $37^{\circ} \mathrm{C}$ for 1 day, and DNA content was assessed by flow cytometry. Numbers indicate the percentage of cells at a given cell cycle stage. C) Expression of p21, cyclin D1, LRH-1, and CYP11B1 at 33 or $37^{\circ} \mathrm{C}$ after 1 day. $* P<0.05$; $* * P<0.01$ vs. cells grown at $33^{\circ} \mathrm{C}$. NS, not significant; rel., relative.

\section{CYP11A1 and CYP11B1 are preferentially expressed in a distinct cell cycle stage}

These data suggested that steroidogenic enzymes are expressed in a cell cycle-dependent manner. We therefore aimed to investigate whether CYP11A1 and CYP11B1 are preferentially expressed at a distinct cell cycle stage. The murine crypt cell-like cell line mICcl2 $(5,9,11)$ was treated with nocodazole overnight to synchronize cells in the $G_{2} / M$ phase and then released into the cell cycle. After different time intervals cell cycle progression was monitored, and gene expression was analyzed. As shown in Fig. 3A, most cells accumulated in the $\mathrm{G}_{2} / \mathrm{M}$ stage after nocodazole treatment, but gradually returned into the cell cycle after removal of the inhibitor. Analysis of mRNA expression revealed that nocodazole-treated cells had increased levels of cyclin B1 (a $\mathrm{G}_{2} / \mathrm{M}$ cyclin), which further increased for the next $4 \mathrm{~h}$ and then decreased (Fig. $3 B$ ). In contrast, cyclin D1 (a G $\mathrm{G}_{1} / \mathrm{S}$ cyclin) mRNA levels were lower in nocodazole-treated cells and only increased after $12 \mathrm{~h}$, when most cells had reached the $\mathrm{G}_{1} / \mathrm{S}$ transition. Interestingly, cyclin $\mathrm{D} 1$ as well as CYP11A1 and CYP11B1 are LRH-1 target genes $(5,7,9)$. Accordingly, CYP11A1 and CYP11B1 mRNA expression showed pattern very similar to that of cyclin D1, with reduced expression in the $\mathrm{G}_{2} / \mathrm{M}$ phase and increased expression in the $G_{1} / S$ phase. In marked contrast, the expression of LRH-1 remained relatively stable during this cell cycle release experiment, apart from a transient increase after $2 \mathrm{~h}$ (Fig. 3B).
Cell cycle inhibition blocks CYP11A1 and CYP11B1 promoter activity

We next assessed whether inhibition of cell cycle progression by various inhibitors would result in reduced steroidogenic enzyme expression. We have previously shown that overexpression of LRH-1 strongly increases CYP11A1 and CYP11B1 promoter activity $(5,9)$. Therefore, the effect of cell cycle blockers was tested on basal and LRH-1-driven CYP11A1 and CYP11B1 promoter activity in mICc12 cells. Treatment of control or LRH-1-transfected mICcl2 cells with different cell cycle blockers induced cell arrest at distinct cell cycle stages. As an example, doxorubicin-treated cells, arresting in $\mathrm{G}_{0}$ and $\mathrm{G}_{2} / \mathrm{M}$ (22), and nocodazole-treated cells, accumulating in the $\mathrm{G}_{2} / \mathrm{M}$ phase, are shown (Fig. 4A) (17). Doxorubicin dose dependently inhibited LRH-1-driven CYP11A1 and CYP11B1 promoter activity and also reduced basal promoter activities. Similarly, in the presence of LRH-1 dose-dependent inhibition of CYP11A1 and CYP11B1 promoter activity could be observed after treatment of cells with nocodazole (inhibitor of mitotic spindle formation), aphidicolin (inhibitor of DNA replication), forskolin (increase in cAMP), and olomoucine (inhibitor of Cdk1 activity) (Fig. 4B). In contrast, no inhibition was observed with the inactive control compound iso-olomoucine (data not shown).

\section{Inhibition of cell cycle blocks LRH-1-induced corticosterone synthesis}

The inhibition of CYP11A1 and CYP11B1 promoter activity suggested that the inhibition of the cell cycle would also block corticosterone production. mICcl2 cells were thus transfected with control or LRH-1 expression vector and treated with various cell cycle blockers, and corticosterone production was assessed. Interestingly, although overexpression of LRH-1 induced the synthesis and release of corticosterone, treatment with doxorubicin, nocodazole, aphidicolin, and olomoucine efficiently blocked LRH-1-mediated corticosterone production (Fig. 4C). These data indicate that in the intestine cell cycle blockers impair extra-adrenal glucocorticoid synthesis through inhibition of CYP11A1 and CYP1B1 promoter activity.

\section{Role of Cdk1 and pRb in LRH-1-induced steroidogenesis}

Cdks are important regulators of cell cycle progression. Olomoucine is a potent inhibitor of Cdk1 (17), and the olomoucine-induced inhibition of LRH-1-driven CYP11A1 and CYP11B1 promoter activity suggested a critical role of Cdk1 in the regulation of intestinal steroidogenesis. As pharmacological inhibitors can have a variety of off-target effects, we further investigated whether inhibition of Cdk1 by overexpression of dominant negative Cdk1 could result in reduced promoter activity. Consistent with previous findings transient transfection of mICcl2 cells with a dominant negative Cdk1 expression plasmid caused a profound inhibition of LRH-1-induced CYP11A1 and CYP11B1 activity (Fig. 5A, B). In contrast, no such inhibition was observed with wild-type Cdk1. 

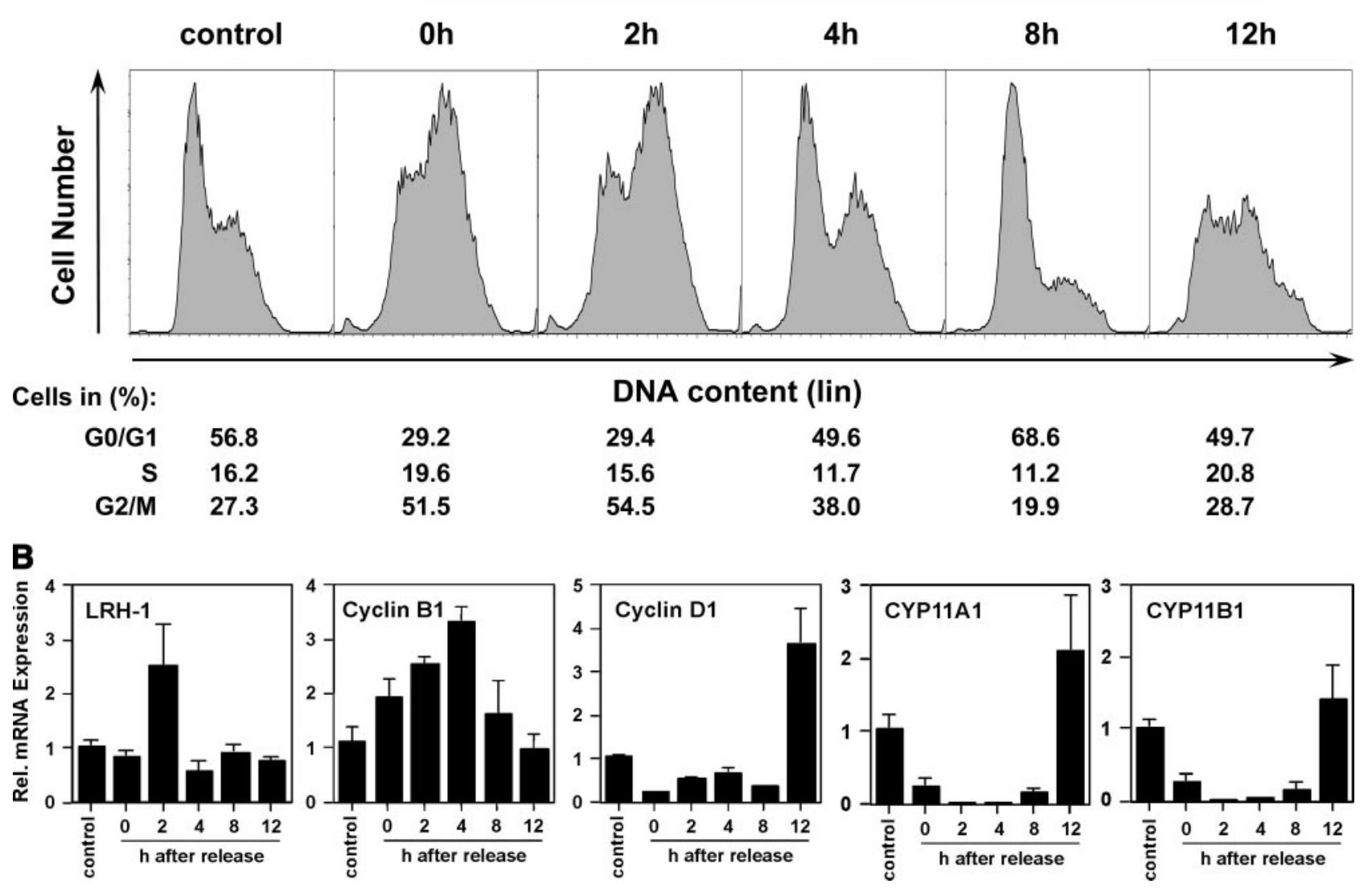

Figure 3. Steroidogenic enzymes are expressed at distinct cell cycle stages. A) mICcl2 cells were synchronized with nocodazole overnight and then were released into the cell cycle for the time intervals indicated. DNA profiles at given time points (hours after release) are shown. The percentage of cells in given cell cycle stages is indicated. $B$ ) Cells were collected at different time points after release from nocodazole block, and the expression of LRH-1, cyclin B1, cyclin D1, CYP11A1 and CYP11B1 was measured by real-time PCR. A typical experiment of three is shown. Rel., relative.

$\mathrm{pRb}$ plays a critical role in the regulation of cell cycle progression (23). In the resting cell $\mathrm{pRb}$ binds and inactivates the transcription factor E2F. Mitogenic stimuli lead to the activation of Cdks, which then phosphorylate and inactivate $\mathrm{pRb}$, releasing $\mathrm{E} 2 \mathrm{~F}$ and thereby promoting cell cycle entry. Because of its prominent role in cell cycle regulation, we tested the effect of $\mathrm{pRb}$ overexpression on LRH-1-driven steroidogenic enzyme expression. In agreement with the above-described results we found that increased levels of $\mathrm{pRb}$ profoundly inhibited CYP11A1 and CYP11B1 induction (Fig. $5 C, D$ ). These data clearly show that inhibition of cell cycle progression results in reduced CYP11A1 and CYP11B1 promoter activity.

\section{Cell cycle inhibitors do not affect LRH-1 nuclear localization}

Many nuclear receptors are cytoplasmic in their inactive stage but translocate to the nucleus after ligand binding, e.g., the glucocorticoid receptor (24). To investigate whether the inhibitory effect of cell cycle blockers on the (LRH-1-induced) expression of steroidogenic enzymes would be due to inhibition of LRH-1 translocation, we analyzed the nuclear localization of fluorescence-tagged
LRH-1. Untagged and GFP-tagged LRH-1 had comparable CYP11A1 promoter-inducing activities in mICcl2 cells, which were equally inhibited after treatment with aphidicolin or doxorubicin (Fig. 6A). mICcl2 cells were then transfected with GFP-tagged LRH-1, and the intracellular localization after treatment of cells with various cell cycle inhibitors was monitored by fluorescence microscopy. In mICcl2 cells LRH-1 constitutively localized to the nucleus, and its localization was not altered after treatment with doxorubicin or other cell cycle inhibitors (Fig. 6B). As doxorubicin treatment resulted in increased background fluorescence, we also analyzed the localization of untagged LRH-1 protein on doxorubicin treatment by subcellular fractionation. Figure $6 C$ shows that in untreated and doxorubicin-treated cells LRH-1 was only detected in the nuclear fraction and was absent from the cytoplasmic fraction.

\section{Cell cycle inhibitors alter LRH-1 transcriptional activity}

To further address whether the inhibitory effect of cell cycle inhibitors on CYP11A1 and CYP11B1 promoter activity was due to specific inhibition of LRH-1 activity, rather than altering other promoter-activating factors, we 


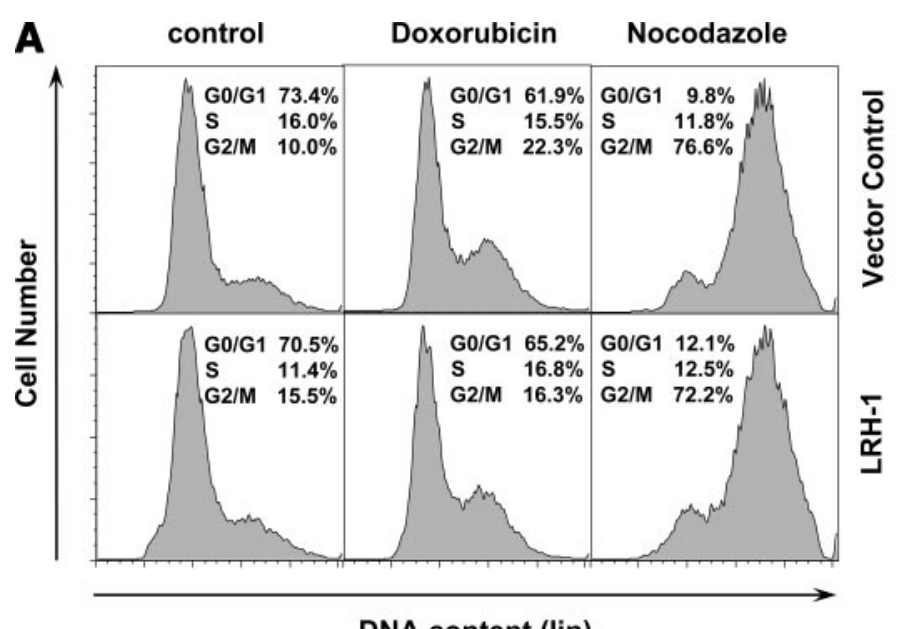

DNA content (lin)
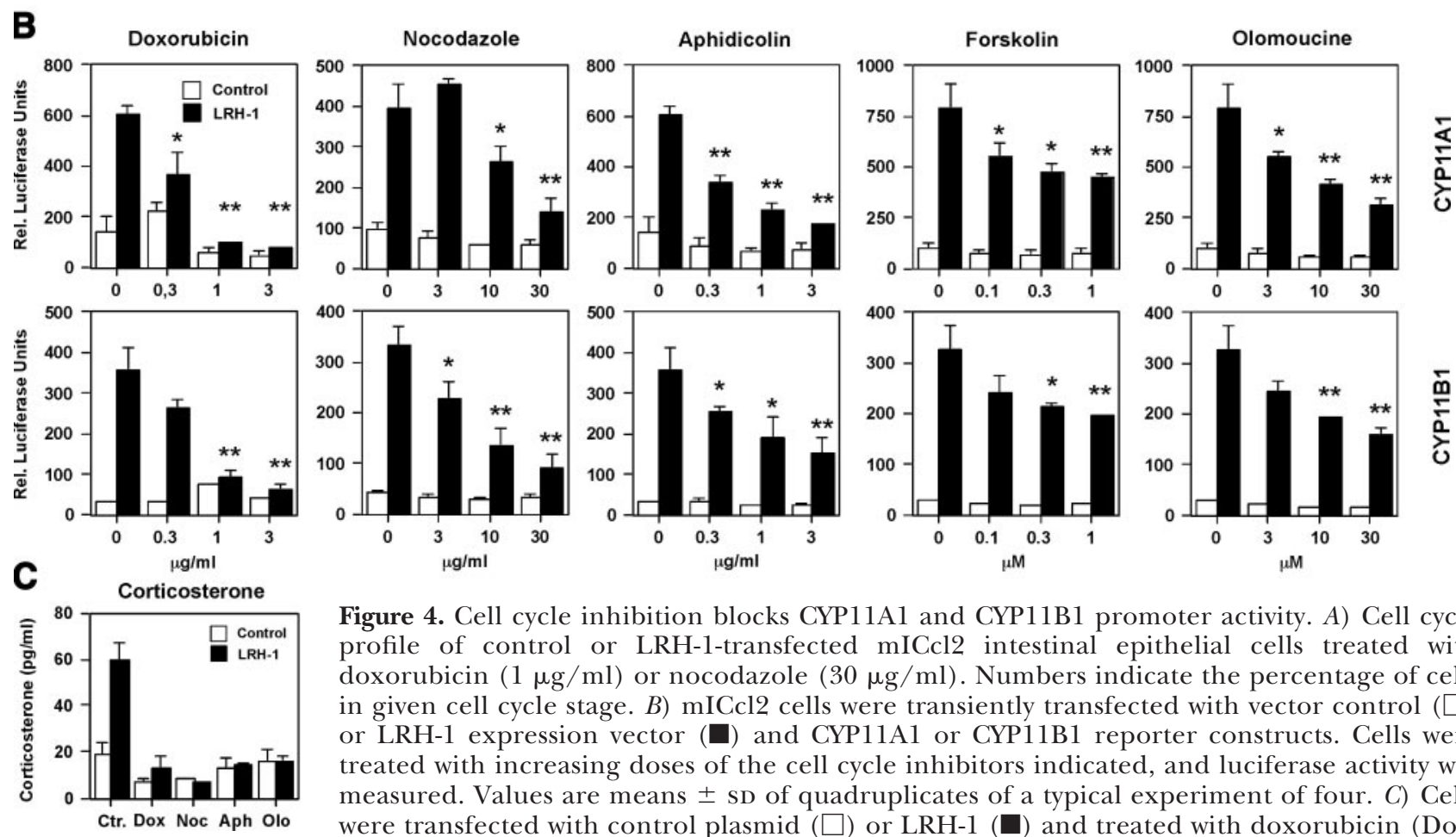

Figure 4. Cell cycle inhibition blocks CYP11A1 and CYP11B1 promoter activity. A) Cell cycle profile of control or LRH-1-transfected mICcl2 intestinal epithelial cells treated with doxorubicin $(1 \mu \mathrm{g} / \mathrm{ml})$ or nocodazole $(30 \mu \mathrm{g} / \mathrm{ml})$. Numbers indicate the percentage of cells in given cell cycle stage. $B$ ) mICcl2 cells were transiently transfected with vector control $(\square)$ or LRH-1 expression vector ( $\boldsymbol{\square})$ and CYP11A1 or CYP11B1 reporter constructs. Cells were treated with increasing doses of the cell cycle inhibitors indicated, and luciferase activity was measured. Values are means \pm SD of quadruplicates of a typical experiment of four. C) Cells were transfected with control plasmid $(\square)$ or LRH-1 $(\square)$ and treated with doxorubicin (Dox, $1 \mu \mathrm{g} / \mathrm{ml}$ ), nocodazole (Noc, $30 \mu \mathrm{g} / \mathrm{ml}$ ), aphidicolin (Aph, $1 \mu \mathrm{g} / \mathrm{ml}$ ), or olomoucine (Olo, 30 $\mu \mathrm{M})$. Corticosterone in the supernatant was measured by RIA. Values are means $\pm \mathrm{SD}$ of triplicates. $* P<0.05 ; * * P<0.01$ vs. control (Ctr.) treated cells. Rel., relative.

transfected cells with a heterologous LRH-1 reporter construct consisting of five LRH-1 response elements (LRH-1 RE 5x) and assessed the effect of cell cycle blockers on LRH-1-induced transcriptional activity (Fig. 7). Transient expression of LRH-1 strongly induced the LRH-1 reporter activity, which was substantially inhibited after treatment of cells with doxorubicin, nocodazole, or aphidicolin. These findings support the notion that LRH-1 transcriptional activity is regulated in a cell cycledependent manner.

\section{DISCUSSION}

We have previously demonstrated a dominant role of LRH-1 in the control of intestinal glucocorticoid synthesis
$(5,9,10)$. Consequently, LRH-1 haploinsufficient mice were found to have reduced intestinal glucocorticoid synthesis (5) and increased susceptibility to development of experimental IBD (10). However, LRH-1 not only regulates steroid synthesis but also controls intestinal epithelial cell proliferation and renewal through the induction of cyclin D1 and E1 expression (7). Thus, LRH-1 may contribute to intestinal epithelial layer integrity via two mechanisms, crypt cell proliferation and epithelial cell renewal on one hand and suppression of intestinal inflammation and regulation of epithelial tight junctions via the induction of local glucocorticoid synthesis on the other hand $(7,10,25)$. In this study, we now show that the relation among LRH-1, the cell cycle, and glucocorticoid synthesis is even more complex and is not 


\section{A}

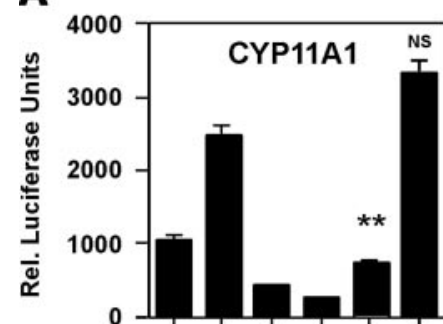

B
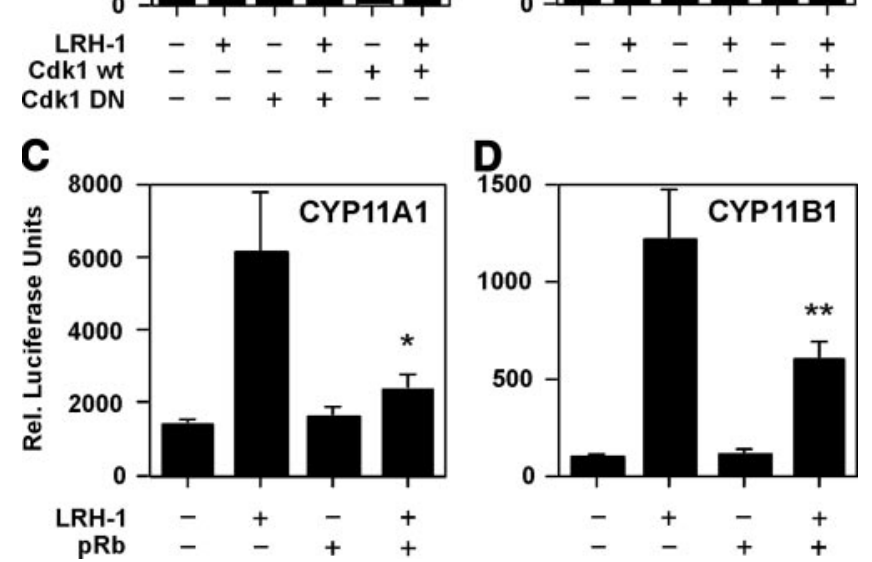

Figure 5. Dominant negative Cdk1 and pRb block steroidogenic enzyme expression. $A, B)$ mICcl2 cells were transfected with LRH-1, wild-type Cdk1, or dominant negative Cdk1 and either CYP11A1 $(A)$ or CYP11B1 $(B)$ reporter constructs. $C, D$ ) Cells were transfected with LRH-1 and pRb as indicated, and CYP11A1 $(C)$ or CYP11B1 $(D)$ induction was measured. Values are means \pm SD of triplicates of a typical experiment. $* P<0.05 ; * * P<0.01$ vs. control treated cells. NS, not significant; rel., relative.

only unidirectional but also extends on cell cycle stagedependent regulation of LRH-1 activity and thereby glucocorticoid synthesis in the intestinal epithelium.

A first hint for cell cycle-dependent control of intestinal glucocorticoid synthesis came from the observation that both LRH-1 and steroidogenic enzymes are predominantly expressed in the proliferating cells of the intestinal crypts $(3,7)$. We confirmed these data by demonstrating that LRH-1, cyclin D1, and CYP11B1 are expressed in the alkaline phosphatase-negative epithelial cells of the small intestinal crypts (Fig. 1A, $B$ ). In addition, we have previously made the surprising finding that an increase in intracellular cAMP levels after treatment of intestinal epithelial cells with forskolin leads to reduced expression of steroidogenic enzymes, whereas forskolin promotes their expression in adrenal-derived cells (9). In this study we now also show that the cell cycle blocker forskolin significantly inhibits LRH-1 activity and LRH-1-induced steroidogenesis in intestinal epithelial cells. This inhibitory effect on LRH-1 activity was not restricted to forskolin but was uniformly observed with a wide variety of cell cycle-modulating compounds and molecules. This suggests that the forskolin-induced inhibition of intestinal steroidogenesis is probably not mediated via the elevation of cAMP and subsequent activation of protein kinase A but rather via inhibition of cell cycle progression. Much in line with this notion is our observation that differentiation of proliferating crypt cell-like YAMC cells to more mature epithelial cells results in cell cycle arrest, as illustrated by reduced proliferation, an increase in p21/Waf expression and reduced D1 expression and a simultaneous reduction in the expression of steroidogenic enzymes. Similarly, we observed that cell cycle synchronization of intestinal epithelial cells leads to a cyclic expression pattern of CYP11A1 and CYP11B1, which correlates with that of cyclin D1, i.e., a peak expression in the $\mathrm{G}_{1} / \mathrm{S}$ phase. As all of these genes are targets of LRH-1, it is tempting to speculate that this cell cycle-dependent effect on the expression of steroidogenic enzymes is mediated via the regulation of LRH-1 activation. Indeed, we have seen that cell cycle arrest by different means blocks the transcriptional activity of LRH-1, whereas it only minimally alters LRH-1 expression levels.

Of particular interest in this regard is a recent observation by Lee et al. (26) that extracellular signal-regulated kinase (ERK) 1/2-mediated phosphorylation of LRH-1 in the hinge region strongly affects its transcriptional activity. ERK1/2 is known to be critically involved in growth factor receptor-induced expression of cell cycle proteins and epithelial cell proliferation. Thus, mitogen-induced activation of the mitogen-activated protein kinase pathway and ERK1/2-mediated phosphorylation of LRH-1 may represent an interesting link between cell cycle stage and LRH-1 activity in intestinal epithelial cells. Another possibility that, however, remains to be confirmed, is that Cdks directly phosphorylate and activate LRH-1. Cdks are serine/threonine kinase and are known to phosphorylate amino acid motifs similar to ERK1/2 [(S/T-P) site]. In addition, there are numerous reports that Cdks phosphorylate and activate various nuclear receptors and/or their cofactors $(27,28)$. For example, it has been shown that Cdk2 phosphorylates the cofactor steroid receptor coactivator (SRC) 1, promotes its interaction with the progesterone receptor, and thereby enhances the transcriptional activity of this nuclear receptor (29). On the other hand, the progesterone receptor is also a direct target of Cdk2-mediated phosphorylation (28). Although these processes are well established for other nuclear receptors and tissues, e.g., the progesterone receptor in the prostate, respectively, in prostate cancer, little is known regarding the intestinal epithelium and nuclear receptors that regulate glucocorticoid synthesis. Here, we provide for the first-time evidence that LRH-1 and extraadrenal glucocorticoid synthesis in the intestinal crypts is regulated in a cell cycle-dependent manner.

An interesting observation in our study was the fact that overexpression of pRb substantially blocked LRH-1-induced CYP11A1 and CYP11B1 promoter activation. $\mathrm{pRb}$ is an important gatekeeper of the cell cycle. Its phosphorylation by $\mathrm{Cdk} 4$ leads to its inactivation and dissociation from E2F and subsequent entry into the cell cycle (23). Although our observations are in line with the fact that (unphosphorylated) $\mathrm{pRb}$ maintains cell cycle arrest and therefore inhibits cell cycle-dependent activation of LRH-1 and steroidogenesis in intestinal epithelial cells, they contrast to some extent with a recent finding that pRb enhances SRC2-dependent activity of other nuclear receptors (30). As SRC1, rather than SRC2, may be critical 

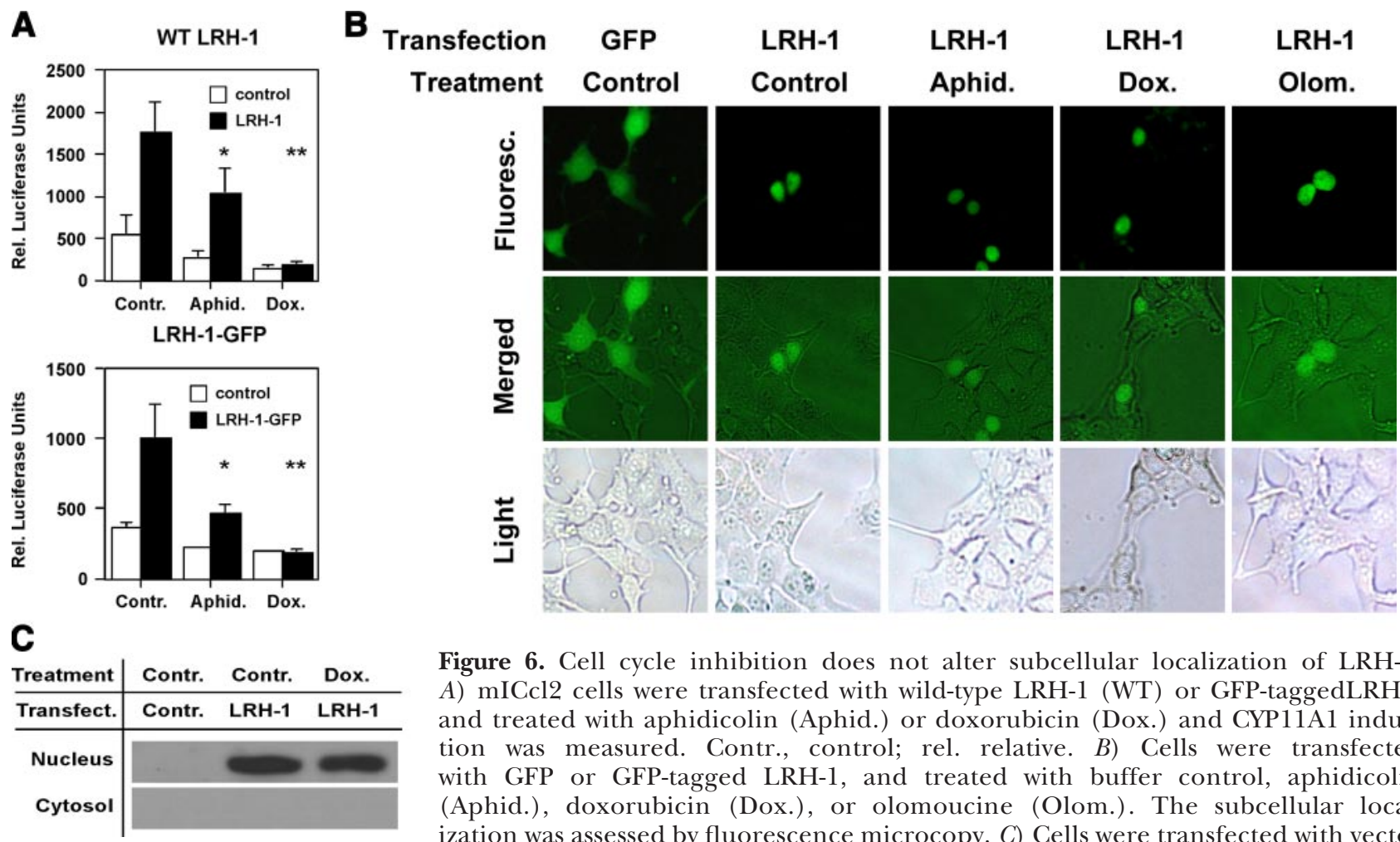

Figure 6. Cell cycle inhibition does not alter subcellular localization of LRH-1. A) mICcl2 cells were transfected with wild-type LRH-1 (WT) or GFP-taggedLRH-1 and treated with aphidicolin (Aphid.) or doxorubicin (Dox.) and CYP11A1 induction was measured. Contr., control; rel. relative. B) Cells were transfected with GFP or GFP-tagged LRH-1, and treated with buffer control, aphidicolin (Aphid.), doxorubicin (Dox.), or olomoucine (Olom.). The subcellular localization was assessed by fluorescence microcopy. C) Cells were transfected with vector control or GFP-tagged LRH-1 and treated with buffer control or doxorubicin. Cytosol and nucleus were separated and the presence of GFP-LRH-1 was detected by Western blot. Rel., relative.

for regulation of LRH-1 activation in intestinal epithelial cells, it is possible that in these cells the dominant effect of $\mathrm{pRb}$ overexpression is the induction of cell cycle arrest (31) and subsequent inhibition of LRH-1 activity.

The question remains as to why LRH-1 activity and associated intestinal glucocorticoid synthesis are regulated in a cell cycle-dependent manner. Possibly, this may allow limitation of expression of steroidogenic enzymes and intestinal glucocorticoid synthesis to the proliferating

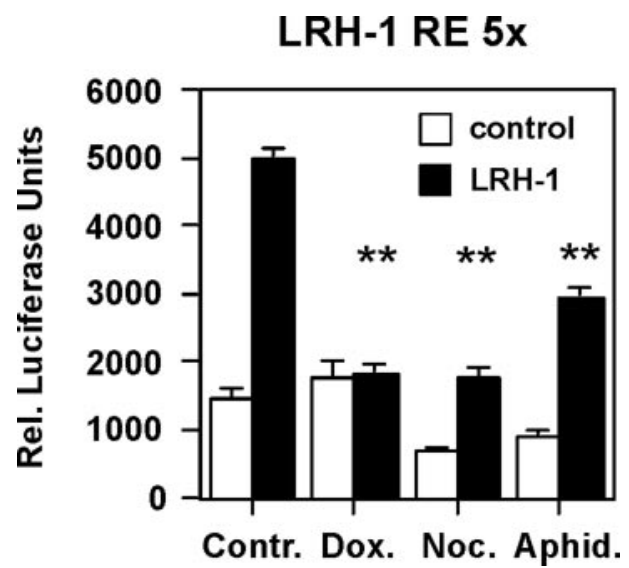

Figure 7. Cell cycle blockers inhibit LRH-1 transcriptional activity. mICcl2 cells were transfected with control vector $(\square)$ or LRH-1 (ם), and a luciferase reporter construct containing 5 LRH-1 response elements. Cells were treated with doxorubicin (Dox.) nocodazole (Noc.), or aphidicolin (Aphid.) and luciferase activity was measured. $* P<0.05$; $* * P<0.01$ vs. control treated cells. Rel., relative. cells of the crypts. Although mature intestinal epithelial cells are short-lived, crypt cells represent a self-renewing and therefore unlimited pool of glucocorticoid-synthesizing cells. This may be important under circumstances in which excessive epithelial cell death takes place (e.g., IBD), and mature epithelial cells could therefore not be a reliable source of immunoregulatory glucocorticoids. Furthermore, damage to the epithelial layer will allow access of intestinal immune cells to luminal antigens, pathogens, and Toll-like receptor ligands, leading to uncontrolled inflammation and tissue damage. Increased proliferation of undifferentiated crypt cells and their differentiation to mature epithelial cells participate in tissue repair, reestablishing an intact intestinal epithelial barrier and thereby limiting intestinal inflammation. The coupling of intestinal glucocorticoid synthesis to cell cycle and crypt cell proliferation may further ensure that intestinal epithelial damage and inflammation are resolved not only by maintaining proper epithelial barrier function but also by suppressing uncontrolled immune cell activation via the synthesis of immunoregulatory glucocorticoids. As LRH-1 is involved in both processes, it probably represents an important gatekeeper of intestinal homeostasis. Much in line with this idea is the observation that LRH-1 is required for intestinal epithelial cell renewal (7) and glucocorticoid synthesis (5) and that LRH-1 deficiency predisposes mice to increased intestinal inflammation (10), probably because of impaired tissue repair and glucocorticoid synthesis. Increased understanding of the concerted processes regulating LRH-1 activation, epithelial proliferation, and intestinal glucocorticoid synthesis may 
have important implications in the development of novel therapies for the treatment of IBD.

The authors thank Seamus Martin (Trinity College, Dublin, Ireland), Robert Whitehead (Vanderbilt University, Nashville, TN, USA), and Ed Harlow (Massachusetts General Hospital Cancer Center, Charlestown, MA, USA) for valuable reagents, and the members of the Brunner Laboratory for helpful advice and input. This work was supported by grants from the Swiss National Science Foundation, the Crohn's and Colitis Foundation of America, and the Bernese Cancer League to T.B.

\section{REFERENCES}

1. Farrell, R. J., and Kelleher, D. (2003) Glucocorticoid resistance in inflammatory bowel disease. J. Endocrinol. 178, 339-346

2. Gonzalo, J. A., Gonzalez-Garcia, A., Martinez C., and Kroemer, G. (1993) Glucocorticoid-mediated control of the activation and clonal deletion of peripheral $\mathrm{T}$ cells in vivo. J. Exp. Med. 177, $1239-1246$

3. Cima, I., Corazza, N., Dick, B., Fuhrer, A., Herren, S., Jakob, S., Ayuni, E., Mueller, C., and Brunner, T. (2004) Intestinal epithelial cells synthesize glucocorticoids and regulate $\mathrm{T}$ cell activation. J. Exp. Med. 200, 1635-1646

4. Parker, K. L., Rice, D. A., Lala, D. S., Ikeda, Y., Luo, X., Wong, M., Bakke, M., Zhao, L., Frigeri, C., Hanley, N. A., Stallings, N., and Schimmer, B. P. (2002) Steroidogenic factor 1: an essential mediator of endocrine development. Recent Prog. Horm. Res. 57, 19-36

5. Mueller, M., Cima, I., Noti, M., Fuhrer, A., Jakob, S., Dubuquoy, L., Schoonjans, K., and Brunner, T. (2006) The nuclear receptor LRH-1 critically regulates extra-adrenal glucocorticoid synthesis in the intestine. J. Exp. Med. 203, 2057-2062

6. Schoonjans, K., Dubuquoy, L., Mebis, J., Fayard, E., Wendling, O., Haby, C., Geboes, K., and Auwerx, J. (2005) Liver receptor homolog 1 contributes to intestinal tumor formation through effects on cell cycle and inflammation. Proc. Natl. Acad. Sci. U. S. A. 102, 2058-2062

7. Botrugno, O. A., Fayard, E., Annicotte, J. S., Haby, C., Brennan, T., Wendling, O., Tanaka, T., Kodama, T., Thomas, W., Auwerx, J., and Schoonjans, K. (2004) Synergy between LRH-1 and $\beta$-catenin induces G1 cyclin-mediated cell proliferation. Mol. Cell 15, 499-509

8. Mueller, C., Corazza, N., Trachsel-Loseth, S., Eugster, H. P., Buhler-Jungo, M., Brunner, T., and Imboden, M. A. (1999) Noncleavable transmembrane mouse tumor necrosis factor- $\alpha$ $(\mathrm{TNF} \alpha)$ mediates effects distinct from those of wild-type TNF $\alpha$ in vitro and in vivo. J. Biol. Chem. 274, 38112-38118

9. Mueller, M., Atanasov, A., Cima, I., Corazza, N., Schoonjans, K., and Brunner, T. (2007) Differential regulation of glucocorticoid synthesis in murine intestinal epithelial versus adrenocortical cell lines. Endocrinology 148, 1445-1453

10. Coste, A., Dubuquoy, L., Barnouin, R., Annicotte, J. S., Magnier, B., Notti, M., Corazza, N., Antal, M. C., Metzger, D., Desreumaux, P., Brunner, T., Auwerx, J., and Schoonjans, K. (2007) LRH-1-mediated glucocorticoid synthesis in enterocytes protects against inflammatory bowel disease. Proc. Natl. Acad. Sci. U. S. A. 104, 13098-13103

11. Bens, M., Bogdanova, A., Cluzeaud, F., Miquerol, L., Kerneis, S., Kraehenbuhl, J. P., Kahn, A., Pringault, E., and Vandewalle, A. (1996) Transimmortalized mouse intestinal cells (m-ICc12) that maintain a crypt phenotype. Am. J. Physiol. 270, C1666-C1674

12. Lu, T. T., Makishima, M., Repa, J. J., Schoonjans, K., Kerr, T. A., Auwerx, J., and Mangelsdorf, D. J. (2000) Molecular basis for feedback regulation of bile acid synthesis by nuclear receptors. Mol. Cell 6, 507-515

13. Schoonjans, K., Annicotte, J. S., Huby, T., Botrugno, O. A., Fayard, E., Ueda, Y., Chapman, J., and Auwerx, J. (2002) Liver receptor homolog 1 controls the expression of the scavenger receptor class B type I. EMBO Rep. 3, 1181-1187

14. Tan, X., Martin, S. J., Green, D. R., and Wang, J. Y. (1997) Degradation of retinoblastoma protein in tumor necrosis factorand CD95-induced cell death. J. Biol. Chem. 272, 9613-9616

15. Van den Heuvel, S., and Harlow, E. (1993) Distinct roles for cyclin-dependent kinases in cell cycle control. Science 262, $2050-2054$

16. Kasibhatla, S., Brunner, T., Genestier, L., Echeverri, F., Mahboubi, A., and Green, D. R. (1998) DNA damaging agents induce expression of Fas ligand and subsequent apoptosis in $\mathrm{T}$ lymphocytes via the activation of NF-KB and AP-1. Mol. Cell 1, 543-551

17. Torgler, R., Jakob, S., Ontsouka, E., Nachbur, U., Mueller, C., Green, D. R., and Brunner, T. (2004) Regulation of activation-induced Fas (CD95/Apo-1) ligand expression in T cells by the cyclin B1/Cdk1 complex. J. Biol. Chem. 279, 3733437342

18. Stasik, I., Rapak, A., Kalas, W., Ziolo, E., and Strzadala, L. (2007) Ionomycin-induced apoptosis of thymocytes is independent of Nur77 NBRE or NurRE binding, but is accompanied by Nur77 mitochondrial targeting. Biochim. Biophys. Acta 1773, 1483-1490

19. Nachbur, U., Kassahn, D., Yousefi, S., Legler, D. F., and Brunner, T. (2006) Posttranscriptional regulation of Fas (CD95) ligand killing activity by lipid rafts. Blood 107, 2790-2796

20. Whitehead, R. H., VanEeden, P. E., Noble, M. D., Ataliotis, P., and Jat, P. S. (1993) Establishment of conditionally immortalized epithelial cell lines from both colon and small intestine of adult H-2Kb-tsA58 transgenic mice. Proc. Natl. Acad. Sci. U. S. A. 90, 587-591

21. Mosmann, T. (1983) Rapid colorimetric assay for cellular growth and survival: application to proliferation and cytotoxicity assays. J. Immunol. Methods 65, 55-63

22. Siu, W. Y., Yam, C. H., and Poon, R. Y. (1999) G1 versus G2 cell cycle arrest after Adriamycin-induced damage in mouse Swiss3T3 cells. FEBS Lett. 461, 299-305

23. Giacinti, C., and Giordano, A. (2006) RB and cell cycle progression. Oncogene 25, 5220-5227

24. Cole, T. J. (2006) Glucocorticoid action and the development of selective glucocorticoid receptor ligands. Biotechnol. Annu. Rev. 12, 269-300

25. Boivin, M. A., Ye, D., Kennedy, J. C., Al-Sadi, R., Shepela, C., and Ma, T. Y. (2007) Mechanism of glucocorticoid regulation of the intestinal tight junction barrier. Am. J. Physiol. Gastrointest. Liver Physiol. 292, G590-G598

26. Lee, Y. K., Choi, Y. H., Chua, S., Park, Y. J., and Moore, D. D. (2006) Phosphorylation of the hinge domain of the nuclear hormone receptor LRH-1 stimulates transactivation. J. Biol. Chem. 281, 7850-7855

27. Wu, R. C., Smith, C. L., and O'Malley, B. W. (2005) Transcriptional regulation by steroid receptor coactivator phosphorylation. Endocr. Rev. 26, 393-399

28. Lange, C. A. (2004) Making sense of cross-talk between steroid hormone receptors and intracellular signaling pathways: who will have the last word? Mol. Endocrinol. 18, 269-278

29. Narayanan, R., Adigun, A. A., Edwards, D. P., and Weigel, N. L. (2005) Cyclin-dependent kinase activity is required for progesterone receptor function: novel role for cyclin A/Cdk2 as a progesterone receptor coactivator. Mol. Cell. Biol. 25, 264-277

30. Batsche, E., Desroches, J., Bilodeau, S., Gauthier, Y., and Drouin, J. (2005) Rb enhances p160/SRC coactivator-dependent activity of nuclear receptors and hormone responsiveness. J. Biol. Chem. 280, 19746-19756

31. Helin, K., Holm, K., Niebuhr, A., Eiberg, H., Tommerup, N., Hougaard, S., Poulsen, H. S., Spang-Thomsen, M., and Norgaard, P. (1997) Loss of the retinoblastoma protein-related p130 protein in small cell lung carcinoma. Proc. Natl. Acad. Sci. U. S. A. 94, 6933- 